\title{
Maturidade sexual do caranguejo Armases rubripes (Rathbun) (Crustacea, Brachyura, Sesarmidae) na Baía de Sepetiba, Rio de Janeiro, Brasil
}

\author{
Giovana V. Lima ${ }^{1} \&$ Lídia M. Y. Oshiro ${ }^{2}$ \\ 1 Programa de Pós-graduação em Biologia Animal, Universidade Federal Rural do Rio de Janeiro. \\ 2 Estação de Biologia Marinha, Universidade Federal Rural do Rio de Janeiro. Rua Sereder, Itacuruçá, 23880-000 \\ Mangaratiba, Rio de Janeiro, Brasil. E-mail: oshiro@ufrrj.br
}

\begin{abstract}
Sexual maturity of the crab Armases rubripes (Rathbun) (Crustacea, Brachyura, Sesarmidae) in Sepetiba Bay, Rio de Janeiro, Brazil. The present study analyzed the sexual maturity of Armases rubripes (Rathbun, 1897) in three populations from different environmental conditions (river, beach and mangrove) in the southeastern Brazilian coast. Crabs were sampled monthly from July 2002 to February 2004; they were captured with sieves at Sahy River, during the high tides, and by hand at beach and mangrove areas, during low tides. Animals were captured by establishing a catch effort of two collectors during 15 minutes for each station. At laboratory, all individuals were sexed and the carapace width measured. The gonads, gonopods and abdomen morphology were observed according to shape and macroscopic aspects. A total of 4,051 specimens were sampled $(1,067$ males, 1,563 females and 1,421 juveniles). There were recognized five morphotypes: juveniles, immature males, immature females, mature males and mature females. The onset of physiological and morphological sexual maturity was asynchronous within sexes, of which, males reaching the ability to produce gametes earlier than females. The size at the onset of sexual maturity was significantly different among populations different coming from areas, except for mangrove area. The individuals obtained from mangrove showed the shortest size at the onset of sexual maturity. Therefore, estimates of males and females sizes at morphological sexual maturity the allometric growth analyses are inadequate in this species.
\end{abstract}

KEY WORDS. Ecosystems; gonadal development; Grapsoidea; habitats; morphotypes.

RESUMO. Objetivou-se analisar a maturidade sexual de Armases rubripes (Rathbun, 1897) em três diferentes ambientes (rio, praia arenosa e manguezal) no sudeste do Brasil. Os caranguejos foram coletados mensalmente de julho/2002 a fevereiro/2004, utilizando-se peneiras no Rio Sahy durante as marés altas e, manualmente, na praia e no manguezal, durante as marés baixas. Os animais foram capturados por dois coletores num esforço de 15 minutos por estação de coleta. No laboratório, os exemplares foram separados por sexo e mensurados em relação a largura da carapaça, do abdome e comprimento do gonopódio. As gônadas, gonopódios e o abdome foram analisados segundo sua forma e seu aspecto. Um total de 4.051 indivíduos foram amostrados (1.067 machos, 1.563 fêmeas e 1.421 juvenis). Foram determinados cinco morfotipos: juvenis, machos imaturos, fêmeas imaturas, machos maduros e fêmeas maduras. $\mathrm{O}$ início da maturidade sexual fisiológica e morfológica ocorreu de forma assíncrona, com machos produzindo gametas mais cedo que as fêmeas. O tamanho da primeira maturidade sexual apresentou diferença significativa entre os ambientes analisados, exceção observada para o manguezal. Os indivíduos obtidos no manguezal apresentaram menores tamanhos para o início da maturidade sexual. Embora, estimados os tamanhos da maturidade sexual morfológica de machos e fêmeas, a análise do crescimento alométrico foi inadequado para a espécie.

PALAVRAS-CHAVES. Ecossistemas; desenvolvimento gonadal; Grapsoidea; habitats; morfotipos.

A adaptação à vida terrestre permitiu observar entre os crustáceos braquiúros, uma série de padrões ao longo do ciclo de vida, que pode variar de espécie para espécie, maximizando sua sobrevivência em diferentes circunstâncias (HARTNOLL 1985, Hartnoll \& Gould 1988). Estas adaptações, seguidas de uma ampla diversidade no número de estratégias reprodutivas, podem estar relacionadas aos diferentes nichos (ABELE 1972, ABELE \& MeAns 1977, NG \& TAN 1995, OmoRI et al. 1997) ou aos diferentes fatores abióticos observados (SASTRY 1983).

Os Grapsoidea representam o grupo de maior êxito den-

Revista Brasileira de Zoologia 23 (4): 1078-1086, dezembro 2006 
tre os Brachyura, em termos de colonização de diferentes habitats, podendo ser encontrados nos ambientes marinho, região entremarés de costões rochosos, no estuário, na água doce e associado à bromeliáceas (HARTNOLL 1964, Melo 1996, Abele \& Means 1977, Abele 1992, Diesel 1992, Anger 1995, Diesel \& HORST 1995, TEIXEIRA \& SÁ 1998).

Armases rubripes (Rathbun, 1897) é um caranguejo sesarmídeo de pequeno porte encontrado na zona tropical e subtropical, nas Américas Central e do Sul, de Nicarágua ao Rio de la Plata (CApítol et al. 1977, Melo 1996). No Brasil, é comumente encontrada na região Sul em pântanos salgados, marismas ou manguezais, habitando raízes e bases dos caules da vegetação halófita de gramíneas, como Spartina sp., fissuras e cavidades do substrato. Na região Sudeste, além dos manguezais, são também encontrados em restingas e entre as axilas de bromeliáceas (CApítoli et al. 1977, Fischer et al.1997, Teixeira \& Sá 1998).

O estudo da maturidade sexual representa um importante tópico dentro da biologia, uma vez que permite entender diferentes estratégias adaptativas empregadas pelo organismo e o momento em que machos e fêmeas começam a atuar diretamente na flutuação populacional. Segundo MANTELATTo \& Fransozo (1996), a maturidade pode ser observada através das mudanças ocorridas entre indivíduos juvenis ou imaturos, que deixam essa condição e passam a apresentar habilidades na produção de gametas e para realizar a cópula.

A avaliação do tamanho dos indivíduos no momento em que atingem a maturidade tem sido feita de várias formas, baseando-se em análises de maturidade morfológica, através do crescimento relativo; maturidade fisiológica, através de observações macroscópicas das gônadas; e a maturidade funcional, através de observações comportamentais do processo de corte e cópula (Hartnoll 1974, Vazzoler 1996, Pinheiro \& Fransozo 1998, Moura \& Coelho 2004).

No entanto, variações intraespecíficas no tamanho da maturidade sexual nos Brachyura têm sido relatadas tanto local, como geograficamente, estando associada à temperatura e à disponibilidade de alimentos (WENNER et al. 1974, ANNALA et al. 1980, Hines 1982, Diaz \& Conde 1989, Moura \& Coelho 2004). Por outro lado, VERnET-CORnUBERT (1958) observa para o caranguejo Pachygrapsus marmoratus (Fabricius, 1787), variações sazonais durante o desenvolvimento das características sexuais secundárias, verificando-se a regressão abdominal em fêmeas sexualmente maduras após o período de desova. Todavia, estudos demonstram estar este processo restrito ao gênero, uma vez Flores \& Negreiros-Fransozo (1999) também verificaram esse padrão reprodutivo em Pachygrapsus transversus (Gibbes, 1850).

Estudos sobre a maturidade sexual morfológica e fisiológica foram realizados com várias espécies de Crustacea Brachyura, como: Ruffino et al. (1994), Pinheiro \& Fransozo (1998), CAstiglioni $\&$ Santos (2001), Nicolau \& Oshiro (2002), Moura \& Coelho (2004), Castiglioni et al. (2004) e Coвo \& Fransozo (2005). No entanto, quando se compara o início desta atividade biológica em diferentes ambientes, observam-se apenas os trabal hos sobre matu- ridade sexual morfológica realizados por Hines (1989), CONDE \& Díaz (1992), Benetti \& Negreiros-Fransozo (2004), Castiglioni \& Negreiros-Fransozo (2004) e Castiglioni et al. (2004).

O presente estudo, além de estimar o início da maturidade sexual fisiológica e morfológica de machos e fêmeas do caranguejo Armases rubripes (Rathbun, 1897) em diferentes áreas da Baía de Sepetiba; tem por objetivo, verificar se há na espécie observada, variações nas características sexuais secundárias dos indivíduos sexualmente maduros à forma imatura de desenvolvimento.

\section{MATERIAL E MÉTODOS}

Os caranguejos foram coletados mensalmente, de julho/ 2002 a fevereiro/2004, em três diferentes hábitats da Baía de Sepetiba, litoral sul do Estado do Rio de Janeiro. a) Rio Sahy (22ํ5'S, 4401'W): caracterizado por águas lêntica, substrato areno-argiloso, elevada densidade de vegetação marginal e salinidade média de $1,2 \pm 1,7 \%$ o (0,0-4,0\%o); b) Praia do Sahy (2256'S, 4401'W): pontos de coleta localizados na região supralitoral, com influência de ondas durante a maré alta, substrato arenoso, bancos densos de Spartina alterniflora Loisel, 1890 e salinidade média de 21,9 $\pm 10,3 \%$ (7,0-34,0\%o); c) Manguezal de Itacuruçá (225ㄴ'S, 433' W): pontos de coleta localizados na região de entremarés, substrato areno-lodoso, bancos esparsos e pouco densos de S. alterniflora e salinidade média de 17,0 \pm 10,0\% (9,0-24,0\%o).

No Rio Sahy, os caranguejos foram coletados utilizandose peneiras durante os períodos de maré alta. Essa metodologia foi empregada devido a maior densidade de vegetação marginal e nichos de refúgios, como buracos e fissuras, que dificultavam a captura manual dos exemplares e aumentavam os riscos de acidentes com aracnídeos e ofídios. Durante a elevação da maré, cuja maior amplitude observada foi de 1,5 m, os caranguejos eram facilmente capturados passando-se as penei ras através de movimentos rápidos e ascendentes sob a vegetação. $\mathrm{Na}$ praia arenosa e no manguezal, devido a menor densidade desses nichos, os indivíduos foram coletados manualmente durante a maré baixa. Os caranguejos foram capturados por dois coletores num esforço de 15 minutos por estação de coleta. Os animais foram colocados em sacos plásticos, etiquetados e posteriormente congelados. No laboratório, após descongelamento em temperatura ambiente, realizou-se a triagem, a identificação e a separação por sexo.

Após a separação, os indivíduos capturados foram mensurados com auxílio de paquímetro com 0,01 mm de precisão, quanto à largura da carapaça, largura do abdome e comprimento do gonopódio. Posteriormente, os exemplares foram dissecados para análise macroscópica das gônadas, observando-se sua coloração, forma e espessura em relação ao hepatopâncreas e a cavidade cefal otorácica, sendo estas gônadas classificadas em cinco estágios (Tab. I). Os machos capturados foram observados quanto à rigidez, espessura e coloração dos gonopódios; enquanto as fêmeas foram observadas quanto à 
forma e a presença de cerdas nos somitos abdominais e nos pleópodos. A partir destas informações, foram agrupados os indivíduos imaturos e maduros de ambos os sexos, para posterior análise em relação à maturidade sexual morfológica.

A comparação entre os diferentes tamanhos de maturidade sexual observados entre machos e fêmeas, e ao longo dos diferentes hábitats estudados foram realizados utilizando-se a análise de variância (ANOVA dois critérios), ao nível de significância de 0,05.

O tamanho da maturidade sexual fisiológica foi determinada após a distribuição dos indivíduos em classes de tamanho, sendo o tamanho do início da maturidade sexual estimado na freqüência de $50 \%$, onde os indivíduos de cada sexo encontram-se fisiologicamente e morfologicamente maduros (VAZzoler 1996). O mesmo método foi utilizado para comparar a maturidade sexual morfológica.

\section{RESULTADOS}

Foi analisado um total de 4.051 caranguejos, sendo 1.067 machos, 1.563 fêmeas e 1.421 juvenis. Deste total, 2.344 (402 machos, 758 fêmeas e 1.184 juvenis) foram coletados no Rio Sahy; 830 (304 machos, 373 fêmeas e 153 juvenis) na região de supralitoral da praia arenosa e 877 (361 machos, 432 fêmeas e 84 juvenis) no manguezal.

Foram verificados cinco morfotipos para A. rubripes, segundo os diferentes estágios de desenvolvimento (Tab. II).

O tamanho médio e o número das populações de caranguejos variaram nos três ambientes analisados. A praia arenosa representou o ambiente com indivíduos com maior tamanho médio $(8,0 \pm 3,5 \mathrm{~mm})$ e maior amplitude $(1,8-18,0 \mathrm{~mm}) \mathrm{em}$ relação à largura da carapaça, seguida de manguezal $(7,2 \pm 3,1$ $\mathrm{mm}$ e 1,7-17,3 mm) e Rio Sahy (6,3 $\pm 2,4 \mathrm{~mm}$ e 0,1-15,8 mm) (Tab. III). O tamanho médio dos morfotipos apresentou variação nos diferentes ambientes, exceção observada entre os machos imaturos, que não apresentaram diferença significativa $(p>0,05)$. Os machos e fêmeas maduras apresentaram maiores tamanhos na praia arenosa (10,4 $\pm 2,8 \mathrm{~mm}$ e 13,5 $\pm 2,2 \mathrm{~mm}$, respectivamente, $p<0,05)$, enquanto as fêmeas imaturas $(5,6$ $\pm 1,5 \mathrm{~mm}$ ) foram maiores no manguezal ( $p<0,05$ ).

No Rio Sahy, machos e fêmeas apresentaram os diferentes estágios de desenvolvimento gonadal ao longo de todas as classes de tamanho, exceto na classe de 0,1-2,0 mm, onde todos os animais foram juvenis (Fig. 1). Ainda nesta figura, verifica-se que os machos atingiram a maturidade sexual em tamanhos menores quando comparados com as fêmeas, uma vez que se observa a presença de machos com gônadas desovadas ou esgotadas na classe de tamanho entre 6,1-8,0 mm de largura da carapaça. Por outro lado, as fêmeas apresentaram-se nesse estágio somente na classe seguinte, de 8,1-10,0 mm. No entanto, a classe de tamanho de 4,1-6,0 mm representa a fase de transição entre os indivíduos imaturos e maduros, uma vez que $50 \%$ dos indivíduos capturados encontraram-se maduros nas classes subseqüentes, entre 6,1-10,0 mm.

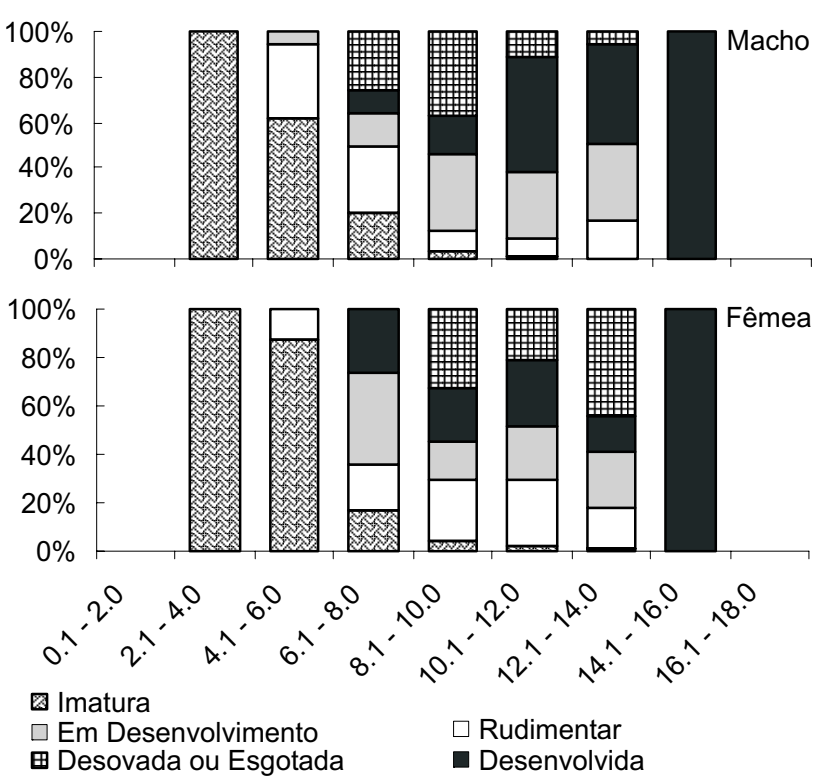

Figura 1. Distribuição por classes de tamanho dos diferentes estágios de desenvolvimento gonadal de machos e fêmeas de A. rubripes capturados no Rio Sahy, Mangaratiba, Rio de Janeiro.

Nas figuras 2 e 3, verifica-se um tamanho de $8,0 \mathrm{~mm}$ para o início da maturidade sexual dos machos enquanto as fêmeas foram observadas a partir de $11,3 \mathrm{~mm}$.

Assim como observado anteriormente, os indivíduos capturados na Praia do Sahy apresentaram diferentes estágios de desenvolvimento gonadal ao longo de todas as classes de tamanho (Fig. 4). Os machos também atingiram a maturidade sexual num tamanho menor queas fêmeas, sendo possível a visualização através da presença de machos com gônadas desovadas ou esgotadas na classe de 6,1-8,0 mm de largura da carapaça, enquanto as fêmeas, de 8,1-10,0 mm. Neste hábitat, o tamanho de transição entre machos imaturos e maduros não ficou tão evidente quanto no rio, observando-se para ambos os sexos, uma transição na classe de $6,1-8,0 \mathrm{~mm}$, com presença de $50 \%$ da população atingindo a maturidade na faixa de 8,1-10,0 mm (Fig. 4).

Na praia, a maturidade sexual fisiológica estimada para machosfoi de 7,5 mm, enquanto para as fêmeas foi de 10,7 mm (Figs 2 e 3).

No manguezal, ao contrário dos hábitats anteriormente mencionados, machos e fêmeas de A. rubripes apresentaram sincronia para o início da maturidade sexual, apresentando gônadas desovadas ou esgotadas na faixa de 4,1-6,0 mm de largura da carapaça e $50 \%$ dos indivíduos coletados na classe de 6,1-8,0 mm (Fig. 5).

Entretanto, machos apresentaram um tamanho de 7,0 mm de largura para o início da maturidade, enquanto para as fêmeas apresentaram um tamanho estimado de 8,0 mm (Figs 2 e 3).

As observações obtidas para a espécie nos diferentes hábitats, permitiram estimar e caracterizar o início da maturi- 

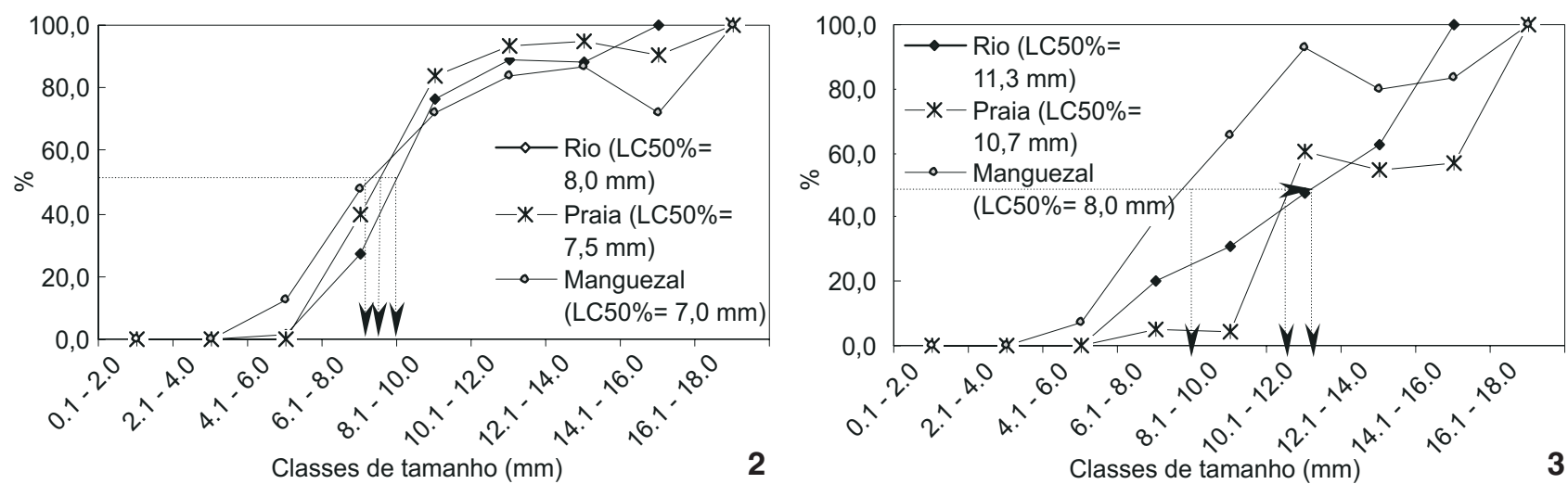

Figuras 2-3. Maturidade sexual fisiológica de machos (2) e de fêmeas (3) de A. rubripes capturados em diferentes ambientes da Baía de Sepetiba, Rio de Janeiro.
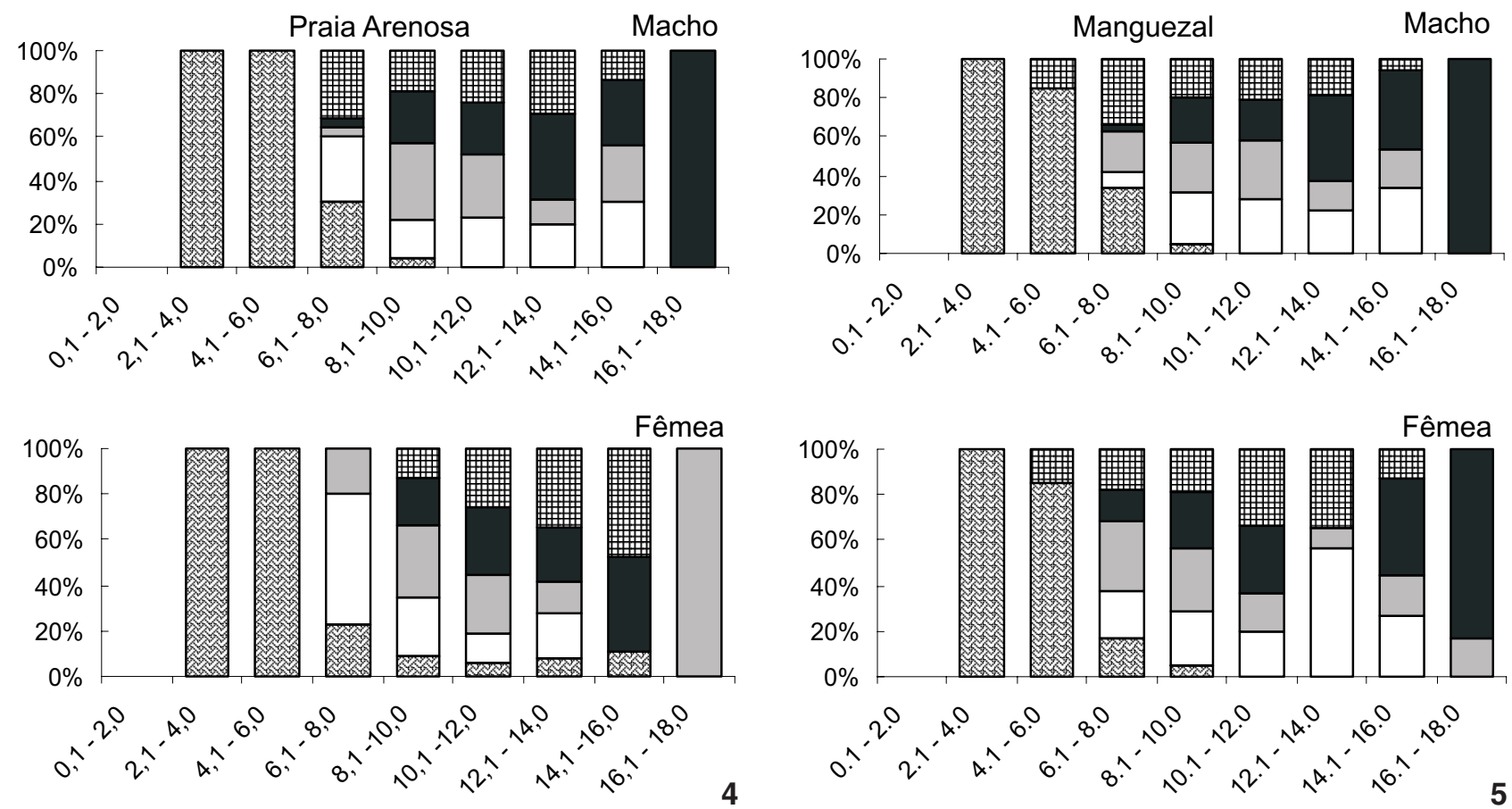

Fêmea

⿴囗⿱一一) Imatura $\square$ Rudimentar $\boxplus$ Desovada ou esgotada $\square$ Em Desenvolvimento $\square$ Desenvolvida

Figuras 4-5. Distribuição por classes de tamanho dos diferentes estágios de desenvolvimento gonadal de machos e fêmeas de A. rubripes capturados na região de entremarés da Praia do Sahy (4) e no manguezal de Itacuruçá, Mangaratiba (5).

dade sexual fisiológica. No entanto, quando observações foram realizadas tomando-se como base as características morfométricas do gonopódio e abdome, verificou-se uma variação dos caracteres entre os diferentes morfotipos (Tab. II).

$\mathrm{Na}$ figura 6, observa-se que o desenvolvimento dos gonopódios em diferentes hábitats ocorre numa classe de tamanho inferior ao estimado para o desenvolvimento do abdome nas fêmeas (Fig. 7).

\section{DISCUSSÃO}

As fêmeas apresentaram maiores tamanhos que os machos no rio e na praia, ocorrendo o inverso no manguezal, assim como observado por CASTIGLIONI et al. (2004) em diferentes manguezais do Estado de São Paulo. De acordo com GherARDI \& Michel (1989) e Abrams (1988), entre os crustáceos, é comum que os machos atinjam os maiores tamanhos, provavelmente 

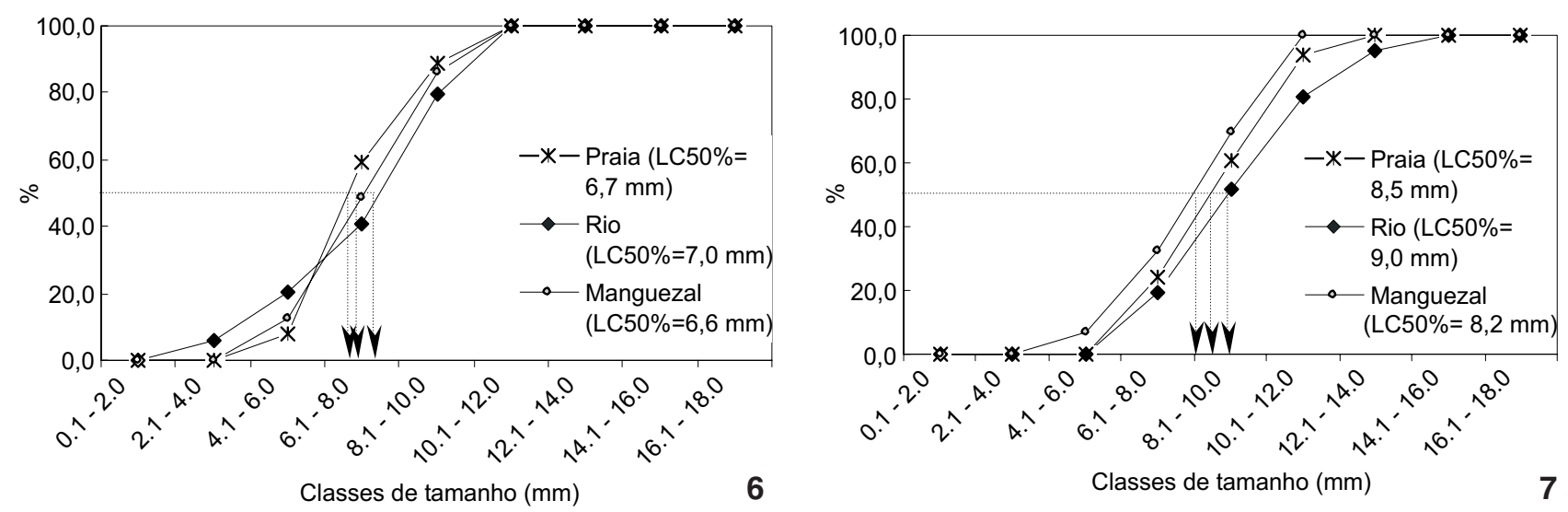

Figuras 6-7. Distribuição por classes de tamanho dos gonopódios (6) e do abdome de fêmeas (7) de A. rubripes em diferentes ambientes da Baía de Sepetiba, Rio de Janeiro.

Tabela I. Caracterização macroscópica dos estágios de maturação gonadal de Armases rubripes, segundo a forma, tamanho, espessura e coloração.

\begin{tabular}{|c|c|c|}
\hline Estágios gonadais & Características dos machos & Características das fêmeas \\
\hline Imaturo & $\begin{array}{l}\text { Gônadas não detectadas sob microscópio } \\
\text { estereoscópio. }\end{array}$ & Gônadas não detectadas sob microscópio estereoscópio. \\
\hline $\begin{array}{l}\text { Intermediário A } \\
\text { (Rudimentar) }\end{array}$ & $\begin{array}{l}\text { Vaso deferente filamentoso e translúcido, } \\
\text { detectável sob microscópio estereoscópio. }\end{array}$ & $\begin{array}{l}\text { Ovário filamentoso e translúcido ou amarelo claro, } \\
\text { detectável sob microscópio estereoscópio. }\end{array}$ \\
\hline $\begin{array}{l}\text { Intermediário B } \\
\text { (Em desenvolvimento) }\end{array}$ & $\begin{array}{l}\text { Vaso deferente em início de enovelamento, } \\
\text { translúcido ou esbranquiçado e ocupando } \\
\text { volume reduzido na cavidade do corpo. }\end{array}$ & $\begin{array}{l}\text { Ovário com forma característica de "H", com cerca de 1/4 } \\
\text { do volume do hepatopâncreas e coloração variando entre } \\
\text { o amarelo, laranja claro e o laranja brilhante. }\end{array}$ \\
\hline Desenvolvido & $\begin{array}{l}\text { Gônadas (testículos + vasos deferentes) ocupando } \\
\text { cerca de } 1 / 4 \text { do volume do hepatopâncreas com } \\
\text { enovelamento completo e coloração branca. }\end{array}$ & $\begin{array}{l}\text { Ovário com lóbulos ocupando cerca de } 1 / 2 \text { do volume do } \\
\text { hepatopâncreas e coloração variando entre o laranja } \\
\text { escuro, marrom e o vinho. }\end{array}$ \\
\hline Desovado ou esgotado & - & $\begin{array}{l}\text { Gônadas não detectadas sob microscópio estereoscópio, } \\
\text { semelhante à condição imatura ou muito filamentosa ou } \\
\text { delgada. }\end{array}$ \\
\hline
\end{tabular}

para assegurar a cópula e garantir a competição intraespecífica. No entanto, um maior crescimento das fêmeas como ocorreu neste estudo, pode maximizar o potencial reprodutivo da espécie, através de uma maior fecundidade (PARKER 1992).

Apesar dos maiores tamanhos observados entre as fêmeas, foi possível verificar nos ambientes analisados uma maturidade fisiológica antecipada entre os machos, assim como observado anteriormente para Sesarma cinereum (Bosc, 1802) (SEIPLE \& SALMON 1987), Goniopsis cruentata (Latreille, 1803) (Сово \& Fransozo 1998), Chasmagnathus granulata Dana, 1851 (LopEz et al. 1997) e Ocypode quadrata (Fabricius, 1787) (HALEY 1969). Possivelmente, estas diferenças durante $\mathrm{o}$ alcance da maturidade podem estar relacionadas à oferta de itens alimentares e estresse ambiental (Wenner et al. 1974, Sastry 1983, Campbell \& Eagles 1983, Stearns \& Koell 1986, Conde \& Díaz 1992, Hines 1989, Negreiros-Fransozo et al. 2003). De acordo com Negreiros-Fransozo et al. (2003), indivíduos dispersos em ambientes com condições extremas, podem começar a procriar antecipadamente se os recursos alimentares mostrarem-se escassos ou com qualidade reduzida.
Para o caranguejo ocypodídeo Uca burgersi Holthis, 1967 estudado por Benetti \& Negreiros-Fransozo (2004), em dois manguezais do Norte do Estado de São Paulo, observa-se diferentes tamanhos para o início da maturidade sexual, atribuindo-se a este processo à diferença na disponibilidade de material orgânico no sedimento (Colpo \& Negreiros-Fransozo 2003).

Conde \& Díaz (1992), analisando fêmeas de Aratus pisonii (H.Milne Edwards, 1837) em diferentes hábitats, também observaram variações intraespecíficas no tamanho da primeira maturidade sexual, verificando-se menores tamanhos ou precocidade dos eventos reprodutivos nas fêmeas dispersas em ambiente com elevado teor de sais dissolvidos. Segundo CONDE et al. (1989), a ocupação de hábitats com maiores valores de salinidade, além de propiciar tamanhos significativamente menores e maturidade antecipada dos indivíduos, induz a um crescimento atrofiado da população, quando comparados com outras populações de locais menos salinos.

Na Baía de Sepetiba, os exemplares de A. rubripes coletados anteciparam a maturidade sexual fisiológica provavelmente 
Tabela II. Caracterização dos morfotipos de Armases rubripes, segundo a forma, espessura, flexibilidade e coloração do gonópodio, a forma e presença de cerdas abdominais e as gônadas.

\begin{tabular}{|c|c|}
\hline Estágios de maturidade sexual & Características morfológicas \\
\hline \multirow[t]{4}{*}{ Juvenil } & Gônadas imaturas (IM) ou rudimentares (RU) \\
\hline & Ausência de pleópodos ou gonopódios \\
\hline & Abdome triangular \\
\hline & Ausência de cerdas externas sobre e nas bordas dos somitos do abdome \\
\hline \multirow[t]{5}{*}{ Macho imaturo } & Gônadas imaturas (IM) ou rudimentares (RU) \\
\hline & Presença de gonopódios vestigiais ou em desenvolvimento \\
\hline & Gonopódios translúcidos, delgados e sem rigidez \\
\hline & Abdome triangular ou em forma de ogiva \\
\hline & Ausência de cerdas externas sobre os somitos do abdome \\
\hline \multirow[t]{5}{*}{ Fêmea imatura } & Gônadas imaturas (IM) ou rudimentares (RU) \\
\hline & Presença de pleópodos vestigiais ou em desenvolvimento \\
\hline & Pleópodos translúcitos, delgados, sem cerdas e sem qualquer rigidez \\
\hline & Abdome triangular ou em forma de ogiva \\
\hline & Ausência de cerdas externas esparsas sobre e nas bordas dos somitos do abdome \\
\hline \multirow[t]{4}{*}{ Macho maduro } & Todos os aspectos gonadais descritos na tabela I \\
\hline & Gonopódios desenvolvidos, opacos (esbranquiçados) e rígidos \\
\hline & $\begin{array}{l}\text { Abdome triangular, porém mais estreito na base de inserção da carapaça do que nos indivíduos juvenis } \\
\text { e imaturos; ou com aspecto de indivíduo imaturo }\end{array}$ \\
\hline & Presença de cerdas esparsas sobre os somitos do abdome \\
\hline \multirow[t]{6}{*}{ Fêmea madura } & Todos os aspectos gonadais descritos na tabela I \\
\hline & Presença de pleópodos desenvolvidos \\
\hline & Pleópodos escuros e com muitas cerdas desenvolvidas \\
\hline & Abdome arredondado, ou em forma de ogiva ou triangular \\
\hline & Presença de cerdas esparsas sobre os somitos e bastante desenvolvidas na borda abdominal \\
\hline & Presença de ovos, quando em período de reprodução \\
\hline
\end{tabular}

devido a uma maior exposição dos indivíduos às águas mais salinas. Concomitantemente, os efeitos de uma maior pressão antrópica ou maior competição interespecífica pelos recursos disponíveis, podem estar levando as populações a procurarem estratégias reprodutivas distintas. No rio, constatou-se uma maturidade sexual tardia, sendo-Ihes atribuído os efeitos de uma reduzida salinidade e à ausência de competição ao longo dos bancos de $\mathrm{S}$. alterniflora.

A maturidade sexual, tanto fisiológica e quanto morfológica, ocorre de forma assíncrona o que pode demonstrar uma importante adaptação reprodutiva da espécie, uma vez que não há limitação reprodutiva.

Durante as mensurações do gonopódio e do abdome para a determinação da maturidade sexual morfológica, foi possível observar variações nos caracteres dos diferentes morfotipos. Segundo HARTNOLL (1974), em várias espécies de Brachyura, estas mudanças morfológicas são observadas principalmente no abdome de fêmeas, podendo ocorrer abruptamente, depois da muda da puberdade, ou aparecer progressivamente depois de várias seqüências de mudas. Segundo HarTnoll $(1974,1982)$ e
Leme (2005), essas modificações anatômicas ocorrem durante o desenvolvimento ontogenético do indivíduo. Todavia, entre os Grapsoidea, estas mudanças morfológicas não são facilmente observáveis uma vez que a largura do abdome de fêmeas imaturas e maduras se sobrepõem, o que dificulta a determinação da maturidade sexual morfológica por métodos de crescimento relativo (HARTNOLL 1965). Essa oscilação, entretanto, ocorreu em ambos os sexos e nos diferentes morfotipos, possibilitando a constatação dessas estruturas corporais tanto nos indivíduos maduros, quanto nos indivíduos juvenis ou imaturos.

Segundo Vernet-Cornubert (1958) e Flores \& NegreirosFransozo (1999), essas variações morfológicas ocorrem nos indivíduos devido ao retorno das estruturas corporais completamente desenvolvidas ao aspecto observado anteriormente nos indivíduos imaturos. Entretanto, foi possível constatar para A. rubripes, assim como mencionado anteriormente, o processo de regressão do abdome; contrapondo-se, porém, a hipótese exclusivista do fenômeno quanto à ocorrência somente entre as fêmeas e entre os representantes do gênero Pachygrapsus. 
Tabela III. Tamanho dos diferentes morfotipos, de acordo com os estágios de maturidade sexual de Armases rubripes ao longo de diferentes ambientes da Baía de Sepetiba, Rio de Janeiro.

\begin{tabular}{|c|c|c|c|c|}
\hline Ambientes & Estágios de maturidade & № de indivíduos & Tamanho médio e desvio padrão (LC) & Amplitude de tamanho ( $\mathrm{mm})$ \\
\hline \multirow[t]{6}{*}{ Rio } & Juvenil & 1184 & $2,2 \pm 0,7$ & $0,1-3,7$ \\
\hline & Macho imaturo & 237 & $5,9 \pm 1,3$ & $3,5-9,8$ \\
\hline & Macho maduro & 165 & $8,8 \pm 2,5$ & $2,5-15,2$ \\
\hline & Fêmea imatura & 655 & $5,3 \pm 1,7$ & $3,2-12,8$ \\
\hline & Fêmea madura & 103 & $9,1 \pm 2,1$ & $6,5-15,8$ \\
\hline & Total & 2344 & $6,3 \pm 2,4$ & $0,1-15,8$ \\
\hline \multirow[t]{6}{*}{ Praia } & Juvenil & 153 & $2,8 \pm 0,4$ & $1,8-3,3$ \\
\hline & Macho imaturo & 107 & $5,6 \pm 1,3$ & $3,8-9,5$ \\
\hline & Macho maduro & 197 & $10,4 \pm 2,8$ & $4,0-18,0$ \\
\hline & Fêmea imatura & 233 & $5,1 \pm 1,5$ & $3,5-10,2$ \\
\hline & Fêmea madura & 140 & $13,5 \pm 2,2$ & $6,7-16,8$ \\
\hline & Total & 830 & $8,0 \pm 3,5$ & $1,8-18,0$ \\
\hline \multirow[t]{6}{*}{ Manguezal } & Juvenil & 84 & $3,1 \pm 0,4$ & $1,7-3,5$ \\
\hline & Macho imaturo & 153 & $5,7 \pm 1,1$ & $3,7-9,5$ \\
\hline & Macho maduro & 208 & $10,0 \pm 2,7$ & $5,0-17,3$ \\
\hline & Fêmea imatura & 271 & $5,6 \pm 1,5$ & $3,7-10,0$ \\
\hline & Fêmea madura & 161 & $9,8 \pm 2,7$ & $4,2-17,2$ \\
\hline & Total & 877 & $7,2 \pm 3,1$ & $1,7-17,3$ \\
\hline
\end{tabular}

\section{AGRADECIMENTOS}

À Fundação de Amparo à Pesquisa do Estado do Rio de Janeiro (FAPERJ) (bolsa de doutorado à primeira autora) e ao estudante Marcelo Rodrigues da Silva Soares.

\section{REFERÊNCIAS BIBLIOGRÁFICAS}

Abele, L.G. 1972. A note on the Brazialian bromeliad crab (Crustacea, Grapsidae). Arquivos de Ciências do Mar, Fortaleza, 12 (2): 123-126.

Abele, L.G. \& D.B. Means. 1977. Sesarma javirsi and Sesarma cookei: Montane terrestrial grapsid crabs in Jamaica. Crustaceana, Leiden, 32 (1): 91-93.

Abrams, P.A. 1988. Sexual difference in resource use in hermit crabs: consequences and causes, p. 283-296. In: G. ChelAzzI $\&$ M. VANNINI (Eds). Behavioral adaptation to intertidal life. Plenum, New York, 524p.

Anger, K. 1995. The conquest of freshwater and land by marine crabs: adaptations in life-history patterns and larval bioenergetics. Journal of Experimental Marine Biology and Ecology, Elsevier, 193: 119-145.

Annala, J.H.; J.L. Mckoy; J.D. Booth \& R.B. Pike. 1980. Size at the onset of sexual maturity in female Jasus edwardsii (Decapoda: Palinuridae) in the New Zeland. Journal of Marine and Freshwater Research, New Zeland, 14 (13): 217-221.
Benetti, A.S. \& M.L. Negreiros-Fransozo. 2004. Relative growth of Uca burgersi (Crustacea, Ocypodidae) from two mangroves in the southeastern Brazilian coast. Iheringia, Série Zoologia, Porto Alegre, 94 (1): 67-72.

Cam pbell, A. \& M.D. Eagles. 1983. Size at maturity and fecundity of rock crabs, Cancer irroratus, from the Bay of Fundy and southwestern Nova Scotia. Fishery Bulletin, Washington, 81 (2): 357-362.

Capítoli, R.R.; C.E. Benvenuti \& N.M. Gianuca. 1977. Ocorrência e observações bioecológicas do caranguejo Metasesarma rubripes (Rathbun) na região estuarina da Lagoa dos Patos. Atlântica, Rio Grande, 2 (1): 50-62.

Castiglioni, D.S. \& M.L. Negreiros-Fransozo. 2004. Comparative analysis of the relative growth of Uca rapax (Smith) (Crustacea, Ocypodidae) from two mangroves in São Paulo, Brazil. Revista Brasileira de Zoologia, Curitiba, 21 (1): 137-144.

Castiglioni, D.S. \& S. Santos. 2001. Reproductive aspects of Cyrtograpsus angulatus Dana, 1851 (Brachyura, Grapsidae) in the Lagoa do Peixe, Rio Grande do Sul State, Brazil. Nauplius, Botucatu, 9 (1): 11-20.

Castiglioni, D.S.; S. Santos; A.L.D. Reigada\& M.L. NegreirosFransozo. 2004. Reproductive ecology of Armases rubripes (Sesarmidae) from mangroves of southeastern Brazil. Nauplius, Botucatu, 12 (2): 109-117.

Совo, V.J. \& A. Fransozo. 1998. Relative growth of Goniopsis

Revista Brasileira de Zoologia 23 (4): 1078-1086, dezembro 2006 
cruentata (Crustacea, Brachyura, Grapsidae), on the Ubatuba Region, São Paulo, Brazil. Iheringia, Série Zoologia, Porto Alegre, 84: 21-28.

CoBo, V. \& A. Fransozo. 2005. Physiological maturity and relationships of growth and reproduction in the red mangrove crab Goniopsis cruentata (Latreille) (Brachyura, Grapsidae) on the coast of São Paulo, Brazil. Revista Brasileira de Zoologia, Curitiba, 22 (1): 219-223.

Colpo, K.D. \& M.L. Negreiros-Fransozo. 2003. Reproductive output of Uca vocator (Herbst, 1804) (Brachyura, Ocypodidae) from three subtropical mangroves in Brazil. Crustaceana, Leiden, 76 (1): 1-11.

Conde, J.E. \& H. Díaz. 1992. Variations in intraspecific relative size at the onset of maturity (Rsom) in Aratus pisonii ( $H$. Milne Edwards, 1837) (Decapoda, Brachyura, Grapsidae). Crustaceana, Leiden, 62 (2): 214-216.

Conde, J.E.; H. DíAz \& G. Rodríguez. 1989. Crecimiento reducido en el cangrejo de mangle Aratus pisonii (H. Milne-Edwards) (Brachyura: Grapsidae). Acta Científica Venezoelana, Venezuela, 40: 159-160.

DiaZ, H. \& J.E. Conde. 1989. Population dynamics and life history of the mangrove crab Aratus pisonii (Brachyura, Grapsidae) in a marine environment. Bulletin of Marine Science, Coral Gables, 45 (1):148-163.

DIESEL, R. 1992. Maternal care in the bromeliad crab, Metapaulias depressus: protection of larvae from predation by danselfly nynphs. Animal Behavior, London, 43: 308-812.

Diesel, R. \& D. Horst. 1995. Breeding in a snail shell: ecology and biology of the Jamaican montane crab Sesarma javirsi (Decapoda: Grapsidae). Journal of Crustacea Biology, Lawrence, 15: 179-195.

Fischer, E.A.; L.F.L. Duarte \& A.C. Araújo. 1997. Consumption of bromeliad flowers by the crab Metasesarma rubripes in a Brazilian coastal forest. Crustaceana, Leiden, 70 (1): 118-120.

Flores, A. \& M.L. Negreiros-Fransozo. 1999. Allometry of the secondary sexual characters of the shore crab Pachygrapsus transversus (Gibbes, 1850) (Brachyura, Grapsidae). Crustaceana, Leiden, 72 (9): 1051-1066.

GherARDI, F. \& F. Michelı. 1989. Relative growth and population structure of the freshwater crab, Potamon potamios palestinensis, in the dead sea area (Israel). Israel Journal of Zoology, Jerusalem, 36: 133-145.

HALEY, S.R. 1969. Relative growth and sexual maturity of the texas ghost crab, Ocypode quadrata ((Fabr.) (Brachyura, Ocypodidae). Crustaceana, Leiden, 17 (3): 285-297.

HARTNOLL, R.G. 1964. The freshwater grapsid crabs of Jamaican. Proceeding of the Linnean Society London, London, 175 (2):145-169.

HARTNOLL, R.G. 1965. Notes on the marine grapsid crab of Jamaica. Proceeding of the Linnean Society of London, London, 17: 113-147.

HARTNOLL, R.G. 1974. Variation in growth pattern between some secondary sexual characters in crabs (Decapoda, Brachyura).
Crustaceana, Leiden, 27 (2): 151-156.

HaRTNoll, R.G. 1982. Growth, p. 111-196. In: D.E. Bliss \& L.G. ABELE (Eds). The biology of Crustacea: embryology, morphology, and genetics. New York, Academic Press, vol. 2, 440p.

HARTNOLL, R.G. 1985. Growth, sexual maturity and reproductive output, p. 101-128. In: A.M. Wenner (Ed.) Factors in adult growth. Rotterdam, Balkema, vol. 3, 362p.

Hartnoll, R.G. \& P.Gould. 1988. Brachyuran life history strategies and the optimization of egg production. Symposia of the Zoological Society of London, Oxford, 59: 1-9.

HINES, A.H. 1982. Allometric constraints and variables of reproductive effort in brachyuran crabs. Marine Biology, Berlin, 69: 309-320.

Hines, A.H. 1989. Geographic variation in size at maturity in brachyuran crabs. Bulletin of Marine Science, Miami, 45 (2): 356-368.

Leme, M.H.A. 2005. Size at sexual maturity of female crabs Sesarma rectum Randall (Crustacea, Brachyura) and ontogenetic variations in the abdomen relative growth. Revista Brasileira de Zoologia, Curitiba, 22 (2): 433-437.

López, L.S.; V.S. Stella \& E.M. Rodríguez. 1997. Size at onset of sexual maturity in Chasmagnatus granulata (Decapoda, Brachyura). Nauplius, Rio Grande, 5 (2): 65-75.

Mantelatto, F.L.M. \& A. Fransozo. 1996. Size at sexual maturity in Callinectes ornatus (Brachyura, Portunidae) from the Ubatuba region (SP), Brazil. Nauplius, Rio Grande. 4: 29-38.

Melo, G.A.S. 1996. Manual de identificação dos Brachyura (caranguejos e siris) do litoral brasileiro. São Paulo, Ed. Plêiade, FAPESP, Museu de Zoologia, 603p.

Moura, N.F.O. \& P.A. Coelho. 2004. Maturidade sexual fisiológica em Goniopsis cruentata (Latreille) (Crustacea, Brachyura, Grapsidae) no estuário do Paripe, Pernambuco, Brasil. Revista Brasileira de Zoologia, Curitiba, 21 (4): 1011-1015.

Negreiros-Fransozo, M.L.; K.D. Colpo \& T.M. Costa. 2003. Allometric growth in the fiddler crab Uca thayeri (Brachyura, Ocypodidae) from a subtropical mangrove. Crustaceana, Leiden, 23 (2): 273-279.

NG, P.K.L. \& C.G.S. TAN. 1995. Geosesarma notophorum sp. nov. (Decapoda, Brachyura, Grapsidae, Sesarminae), a terrestrial crab from Sumatra, with novel brooding behaviour. Crustaceana, Leiden, 68 (3): 390-395.

Nicolau, C.F. \& L.M.Y. OshiRo. 2002. Aspectos reprodutivos do caranguejo Aratus pisonii (H. Milne Edwards) (Crustacea, Decapoda, Grapsidae) do manguezal de Itacuruçá, Rio de Janeiro, Brasil. Revista Brasileira de Zoologia, Curitiba. 19 (Supl. 2): 167-173.

OMORI, K.; K. SHIRAISHI \& M. HARA. 1997. Lifehistories of sympatric mud-flat crabs, Helice japonica and $\mathrm{H}$. tridens (Decapoda, Grapsidae), in a Japanese estuary. Journal of Crustacean Biology, Lawrence, 17 (2): 279-288.

PARKer, G.A. 1992. The evolution of sexual dimorphism in fish. Journal of Fish Biology, London, 41 (b):1-20. 
Pinheiro, M.A.A. \& A. Fransozo. 1998. Sexual maturity of speckled swimming crab Arenaeus cribrarius (Lamarck, 1818) (Decapoda, Brachyura, Portunidae), in the Ubatuba littoral, São Paulo State, Brazil. Crustaceana, Leiden, 71 (6): 15-47.

Ruffino, M.L.; M.D. Telles \& F. D'InCAO. 1994. Reproductive aspects of Chasmagnathus granulata Dana, 1851 (Decapoda, Grapsidae) in the Patos Lagoon estuary - Brazil. Nauplius, Rio Grande, 2: 43-52.

SASTRY, A. 1983. Ecological aspects of reproduction, p 179-269. In: D.E. BuIss (Ed.). The Biology of Crustacea. Academic Press, New York, 383p.

SeIPLE, W.H. \& M. SALmon.1987. Reproductive, growth and lifehistory contrasts between two species of grapsid crabs, Sesarma cinereum and S. reticulatum. Marine Biology, Berlin, 94: 1-6.

Stearns, S.C. \& J.C. Koell. 1986. The evolution of phenotypic plasticity in life-history traits: predictions of reaction norms for age and size at maturity. International Journal of Organic Evolution, Lawrence, 40 (5): 893-913.

TEIXEIRA, R.L. \& H.S. SÁ. 1998. The use of bromeliads as an alternative shelter by the grapsid crab, M etasesarma rubripes (Rathbun, 1897) of southeast Brazil. Boletim do Museu de Biologia Mello-Leitão, Vitória, 8: 13-20.

VAZzoler, A.E.A.M. 1996. Manual de métodos para estudos biológicos de populações de peixes teleósteos: teoria e prática. Maringá, EDUEM, 169p.

Vernet-Cornubert, G. 1958. Recherches sur la sexualité du crabe Pachygrapsus marmoratus (Fabricius). Archives de Zoologie Experimentale et Génerale, Paris, 96: 191-276.

Wenner, A.M.; C. Fusaro \& A.OAten. 1974. Size at onset of sexual maturity and growth rate in crustacean populations. Canadian Journal of Zoology, Ottawa, 52 (9): 1095-1106.

Recebido em 21.X.2005; aceito em 14.X.2006. 